\title{
Using a Lag Reinforcement Schedule to Increase Phonemic Variability in Children With Autism Spectrum Disorders
}

\author{
Kate Koehler-Platten, Saint Cloud State University \\ Laura L. Grow, University of British Columbia \\ Kimberly A. Schulze, Saint Cloud State University \\ Tara Bertone, Autism Matters, Inc.
}

\begin{abstract}
Shaping functional vocal language is difficult when an individual has not yet acquired an echoic repertoire and does not emit sufficient phonemes (i.e., speech sounds) for shaping. Few studies have evaluated interventions to increase the frequency and breadth of phonemes. The current study extended Esch, Esch, and Love (2009) by evaluating the effects of a Lag 1 reinforcement schedule on vocal variability and limiting the definition of variability to responses that incorporated a novel phoneme. For 2 of the 3 participants, the cumulative number of novel phonemes, the percentage of trials with variability, and the number of different phonemes emitted per session increased during the Lag 1 intervention phase.

Key words: echoics, lag schedules, speech, vocal variability
\end{abstract}

Deficits in language skills are a defining characteristic of autism spectrum disorders (ASDs; American Psychiatric Association [DSM-IV-TR], 2000). Language impairments range from severe (e.g., little to no spoken language, stereotyped and repetitive language) to mild (e.g., difficulties with sustaining reciprocal conversations). Some learners with ASD have limited echoic repertoires. An echoic is the term used by Skinner (1957) to identify the verbal operant in which the topography of an individual's verbal behavior has point-to-point correspondence and formal similarity with another individual's verbal behavior. For example, a mother says "ma-ma" and her child repeats

This study was supported in part by the Society for the Advancement of Behavior Analysis Master's Thesis Grant and the Speech Pathology Special Interest Group of the Association for Behavior Analysis International Student Research Grant.

This article is based on a thesis submitted by the first author under the supervision of the second and third authors in partial fulfillment of the requirements for the master's degree in behavior analysis from Saint Cloud State University. We thank committee members John Rapp and Theresa Estrem for their helpful comments.

Correspondence concerning this article may be addressed to: Laura Grow, Educational and Counselling Psychology and Special Education, University of British Columbia, 2125 Main Mall, Vancouver, BC, Canada V6T 1Z4. (e-mail: laura.grow@ubc.ca). the same sounds, "ma-ma." Teaching echoic responses relies on the individual producing a sufficient variety of phonemes. For example, if an individual's vocalizations primarily consist of the sounds "buh" and "oo," an instructor would have difficulty with teaching echoic responses that did not contain those sounds. Thus, sufficient vocal variability is needed to teach a robust echoic repertoire. Deficits in an echoic repertoire in conjunction with limited phonemic variability pose unique challenges for instructors because the intervention recommendations in many of the early intervention curricular manuals (e.g., Leaf \& McEachin, 1999; Lovaas, 2003) rely on an already established echoic repertoire for teaching vocal verbal behavior.

A growing body of research has focused on identifying procedures that increase the frequency and topographical variety of vocal verbal behavior for children with limited vocal verbal skills (e.g., Duker \& van Lent, 1991; Esch, Esch, \& Love, 2009; Lee, McComas, \& Jawor, 2002). Lag schedules have been used to increase response variability for both vocal and nonvocal behaviors (Esch et al., 2009; Lee et al., 2002; Napolitano, Smith, Zarcone, Goodkin, \& McAdam, 2010). Lag schedules of reinforcement are arranged by delivering reinforcers contingent on variation from a certain number of previous responses. For example, 
in a Lag 1 schedule, reinforcers are delivered for responses that are different from an immediately preceding response.

Esch et al. (2009) evaluated a Lag 1 reinforcement schedule for increasing vocal variability for two children who exhibited limited phonemes. The experimenters conducted a 30-min pre-experimental naturalistic observation to identify the number and frequency of phonemes emitted by each participant. During experimental sessions, each trial was initiated by the presentation of a vocal model. Reinforcers were provided for responses that occurred within $5 \mathrm{~s}$ of the model and differed in any way from the previous response. For both participants, the lag schedule resulted in an increase in the frequency of varied vocalizations. The definition used in the Esch et al. study allowed the participant to obtain all possible reinforcers even if participants alternated between two vocalizations (e.g., "ma" and "me"). The number of different phonemes emitted could be as few as two and restricted to those already included in the participants' vocal repertoires. It is unknown to what extent this occurred in the study but the authors noted anecdotally that the children's varied responses tended to be limited to phonemes that required the least amount of tongue repositioning (e.g., "tuh" and "duh" compared to "kuh" and "tee").

Overall, the literature suggests that lag schedules are effective for promoting response variability among responses already in the participant's repertoire. However, little is known about whether lag schedules can be used to generate novel behavior. One notable exception is a study conducted by Lee et al. (2002). Lee and colleagues demonstrated increases in both the percentage of varied responses and the number of novel responses to social questions (i.e., "What do you like to do?" or "How are you?') for 2 of the 3 participants using a Lag 1 schedule. The novel response data provided support that the participants did not simply learn to alternate between two responses; rather, the participants acquired new forms of vocal verbal behavior.

The results of the study by Lee and colleagues (2002) provide preliminary support that in some contexts, lag schedules can be used to generate novel topographies of behavior. The purpose of the current study was to replicate and extend the research by Esch et al. (2009) on the effects of a Lag 1 reinforcement schedule on vocal variability. Specifically, we evaluated the utility of a Lag 1 schedule of reinforcement for (a) promoting variability in phonemes, and (b) increasing the size of the phonemic repertoire.

\section{METHOD}

\section{Participants and Setting}

Three children participated in the study. The participants attended a center providing behavioral early intervention and speech language pathology (SLP) services. Chloe was a 2-year, 10-month old girl diagnosed with autistic disorder. Chloe communicated using a few manual signs (e.g., movie, music) and had no vocal word approximations. Prior to the start of the study, Chloe had mastered "ba" as an echoic response. Chloe attended an early intervention clinic for $30 \mathrm{hrs}$ per week and had been receiving services for 9 months prior to the start of the study.

Ari was a 6-year, 4-month old boy diagnosed with autistic disorder. Ari had no vocal word approximations and no echoic repertoire. At the time of this study, Ari had mastered 11 signs and was working on 9 others as part of his early intervention program. Approximately 20 months prior to this study, Ari was able to vocally mand using a few two-word approximations (e.g., "go eat") and had acquired nine phonemes as echoic responses. He discontinued ABA services for 5 months and, when he returned, he no longer engaged in vocal mands and did not engage in echoic behavior. Additionally, Ari exhibited frequent vocal stereotypy consisting of either rapidly repeating sounds/pairs of sounds or extended highpitched open vowel sounds. Ari's vocal stereotypy was accompanied either by lip movements unnecessary for the production of the sounds or repetitive head shaking. Ari had received $40 \mathrm{hrs}$ of services for 1 year and 8 months prior to his 5-month absence. During the study, Ari attended an early intervention clinic for $30 \mathrm{hrs}$ per week and had been receiving services for 1 year and 3 months after his 5-month absence. 
Lily was a 5-year, 11-month old girl diagnosed with autistic disorder. She had one single word approximation ("mom") and emitted single phonemes ("m," "ah," "oo") as echoic responses. Lily had mastered eight manual signs and was working on six signs as part of her early intervention program. Lily attended an early intervention clinic for $40 \mathrm{hrs}$ per week and had been attending for nine months.

Assessment sessions took place in three different locations at the center (the participant's therapy table, a conference room, and an office space). Baseline and intervention sessions took place in the $3 \mathrm{~m} \times 4 \mathrm{~m}$ office space, which contained a desk, computer, a round table, and chairs. The experimenter sat on the floor in front of and facing the participant who sat either on the floor or on a child-sized chair. The experimenter conducted sessions three to five days each week. During each daily visit, the experimenter conducted either three or four sessions of six trials each.

\section{Response Definitions and Data Collection}

The cumulative number of novel phonemes served as the primary dependent variable, with the number of phonemes within each session and the percentage of trials with variability serving as secondary dependent measures. A phoneme was defined as any of the 40 phonemes included in the English language. A novel phoneme was defined as a phoneme that had not been emitted during any previous experimental session. A varied response was defined as a vocal response that included at least one phoneme not included in the previous response (e.g., "goo" following "gah"). The experimenter used a response coding system to determine whether each emitted phoneme was different from the previous response (D), a repeat response (R), or no response $(\mathrm{N})$. Responses recorded as different were those that varied from the previous response according to the definition of varied phonemes stated previously. A repeat response contained only phonemes that were included in the immediately preceding response, regardless of the order in which they occurred (e.g., "uh buh" following "buh uh"). If a response included at least one of the phonemes included in the model, it was also recorded as echoic (E).

A licensed SLP (the fourth author) served as the second trained observer for interobserver and procedural integrity measures. The experimenter and the SLP independently transcribed all phonemes from videotapes of the sessions in order to collect data on the total number of each of the 40 English phonemes emitted per session and the cumulative number of novel phonemes emitted across experimental sessions. The SLP initially trained the experimenter in the transcription of phonemes. During transcription training, the experimenter and the SLP transcribed videotaped segments of each participant's behavior. Training continued until at least $90 \%$ agreement was achieved for each participant prior to implementing sessions. During initial data collection training, agreement on Ari's behavior was very low (i.e., 60 to $80 \%$ ). The definition of phonemes for Ari was modified to distinguish vocal stereotypy from English phonemes. Thereafter, only phonemes that were emitted after a minimum of $2 \mathrm{~s}$ without stereotypy were recorded for Ari. The SLP trained the experimenter to collect data using transcription for approximately $40 \mathrm{hrs}$ before adequate IOA scores were obtained.

\section{Interobserver Agreement}

Interobserver agreement scores were calculated for (a) responses recorded using the response coding system (i.e., D, R, N, and E) and (b) the transcription of phonemes from videotapes. The SLP independently transcribed phonemes and scored responses using the coding system from videotaped sessions during $31 \%, 31 \%$, and $29 \%$ of sessions for Chloe, Ari, and Lily, respectively. For the response coding system, an agreement was scored if both observers recorded the same code(s) during a given trial. A disagreement was scored if the observers recorded a different code on a given trial. Agreement scores for coding were calculated by dividing the number of agreements by the total number of trials and multiplying by 100 . Agreement scores for coding were $85 \%$ (range $67 \%$ to $100 \%$ ), $88 \%$ (range $67 \%$ to $100 \%$ ), and $84 \%$ (range $33 \%$ to $100 \%$ ) for Chloe, Ari, and Lily, respectively. 
For the post-session transcription from videotapes, an agreement was scored for each phoneme when both observers recorded the same phoneme in the same sequence during the 15-s response interval for each trial. A disagreement was scored when one observer recorded a different phoneme or in a different sequence than the other observer. Agreement scores for the transcription of phonemes were calculated for each session by dividing the number of agreements by the total number of phonemes recorded and multiplying by 100. Agreement scores on phonemes recorded were $85 \%$ (range $71 \%$ to $100 \%$ ), $78 \%$ (range $64 \%$ to $100 \%$ ), and $85 \%$ (range $70 \%$ to $100 \%$ ) for Chloe, Ari, and Lily, respectively. Agreement scores for Ari may have been lower due to the difficulty in distinguishing between phonemes and vocal stereotypy.

\section{Pre-Treatment Assessments}

Naturalistic observation. The experimenter videotaped each participant while she or he played independently and with familiar adults (i.e., their current therapist) for $60 \mathrm{~min}$. No prompts (e.g., say "bah") or other environmental manipulations (e.g., withholding preferred items) were implemented. From the video, the experimenter recorded the frequency of each English-language phoneme emitted by the participant during the observation to determine the frequency and topography of the phonemes emitted in a naturalistic play context. A post-intervention observation was conducted within a month of completing the study, using the same procedures.

$V B-M A P P$. The experimenter conducted the Verbal Behavior Milestones Assessment and Placement Program (VB-MAPP) Level 1 Spontaneous Vocal Behavior subsection and the Early Echoic Skills Assessment (EESA) subtest to assess each participant's vocal verbal repertoire prior to and following intervention (Sundberg, 2008). Level 1 of the Milestone Assessment subtests corresponds to vocal skills that typically developing children acquire by 18 months of age. Using data collected from the 60 -min observation described above, the experimenter determined if each participant demonstrated the skills included in the Spontaneous Vocal
Behavior subsection of the assessment. These skills are: spontaneously emits 5 sounds, spontaneously emits 10 sounds including 5 different sounds, spontaneously emits 25 sounds including 10 different sounds, spontaneously emits 5 different word approximations, and spontaneously emits 15 words or phrases with appropriate intonation and rhythm.

The EESA subtest corresponds to the level 1 Echoic subsection of the VB-MAPP. The EESA tests for a number of different echoic responses, which are divided into five groups. Groups 1 through 3 assess (1) simple and reduplicated sounds, (2) two-syllable combinations, and (3) three-syllable combinations. Groups 4 and 5 assess vocal imitation of emphasis, pitch, and intonation. During the EESA assessment the experimenter presented each vocal model up to three times and recorded whether the participant's closest approximation was correct (1 point), partially correct ( $1 / 2$ point $)$, or incorrect/absent ( 0 points). The experimenter delivered reinforcers following any correct or partially correct echoic responses. Reinforcers were delivered intermittently for attending throughout the assessment to maintain participant engagement when correct responding was minimal.

Echoic assessment. The experimenter probed each of 40 English-language phonemes as an echoic response. Four English phonemes were considered not relevant to the study because they pertained to syllable stress in words. The experimenter presented each consonant or consonant blend in combination with "uh" (e.g., "guh," "thuh") and each vowel singly (e.g., "ah," " ooh") to evaluate each participant's echoic repertoire. The phoneme "uh" was presented in combination with all consonants because many consonants cannot be spoken without a vowel. The phoneme "uh" was chosen in consultation with a licensed SLP (the fourth author). Correct echoic responses that occurred within $5 \mathrm{~s}$ of the experimenter-delivered vocal model resulted in access to preferred items for $30 \mathrm{~s}$ (preference assessment procedure described below). Responses were considered correct if the participant emitted the target response, regardless of whether the response was an exact match to the model. For example, "bahbah" was scored as correct when the model 
"buh", was used because the response included the target phoneme "b." The echoic assessment was completed across multiple six-trial sessions until each of the 40 English phonemes was presented once during the assessment. The echoic assessment was repeated post-intervention to evaluate possible changes in the participants' echoic repertoire following intervention.

\section{Stimulus Preference Assessments}

The experimenter conducted a pairedstimulus preference assessment (PSA) consisting of 16 items to identify a hierarchy of preferred stimuli (Fisher et al., 1992). In an attempt to control for session-by-session fluctuations in preference, the experimenter conducted a brief multiple-stimulus without replacement (MSWO) preference assessment prior to each session using three to five of the preferred items identified by the PSA (DeLeon \& Iwata, 1996). Preferred items identified from the brief MSWO were used as reinforcers during the subsequent session. If the participant signed or gestured for an item/activity that wasn't a top choice in the MSWO or PSA, the experimenter presented both the manded item/ activity and the preferred item identified in the MSWO for $30 \mathrm{~s}$ following eligible responses.

\section{Procedural Integrity}

A trained observer assessed treatment integrity from videotapes for $31 \%, 31 \%$, and $29 \%$ of sessions across all phases for Chloe, Ari, and Lily, respectively. Integrity for each trial was scored as either "yes" (all elements of the trial implemented correctly) or " no" (one or more elements implemented incorrectly). To implement a trial correctly, the experimenter had to gain the participant's attention prior to presenting a vocal model, present the predesignated vocal model within $3 \mathrm{~s}$, and provide a reinforcer within $3 \mathrm{~s}$ of responses eligible for reinforcement. Gaining the participant's attention was defined as the experimenter ensuring that the participant's eye gaze was directed toward the experimenter's face while the vocal model was presented. The experimenter used the participant's name and statements such as "look," or gentle touch (e.g., touching the participant's hand) to facilitate attending. A pre- designated vocal model was defined as the experimenter vocally emitting the specified $\mathrm{CV}$ combination delineated by the session data sheet. Correct provision of a reinforcer was defined as the experimenter providing access to highly preferred reinforcers within $3 \mathrm{~s}$ of a response eligible for reinforcement for 25 to $35 \mathrm{~s}$ (toys or activities) or until edible items were consumed (i.e., swallowed). Treatment integrity scores were calculated by dividing the number of correctly implemented trials by the total number of trials in each session and multiplying the proportion by 100 . Treatment integrity scores were $97 \%, 98 \%$, and $92 \%$ for Chloe, Ari, and Lily, respectively.

\section{Experimental Design and Procedures}

Experimental design. A nonconcurrent multiple-baseline design across participants was used to evaluate the effects of the Lag 1 schedule of reinforcement on vocal variability (Watson \& Workman, 1981). The decision rule for implementing the Lag 1 reinforcement schedule was three consecutive baseline sessions with no novel phonemes.

Vocal models. A set of vocal models was developed in consultation with the SLP and consisted of nine consonant-vowel (CV) combinations from three consonants and three vowels. We chose vocal models according to three criteria: (1) they were easily distinguishable to the listener, and (2) they were among the earliest phonemes mastered by typically developing children, and (3) they were not echoed by the participants during the pre-intervention echoic assessment. Additionally, if more than three consonants or vowels met the criteria, consideration was given to which phonemes, if acquired by the participant, would facilitate the participant's ability to communicate (i.e., the phoneme was included in the names of preferred items or activities).

For each six-trial session, three unique vocal models were presented twice. One vocal model was presented in adjacent trials in each session to determine whether variation in responses was a result of the emergence of echoic control. For example, the vocal models for one session for Lily's evaluation included: goo, hee, goo, too, too, 
Table 1

The first column displays the experimenter-delivered vocal models for each participant. The remaining columns display the phonemes that emerged during intervention, naturalistic observation, and the echoic assessment that corresponded to the vocal models

\begin{tabular}{|c|c|c|c|c|}
\hline Participant & $\begin{array}{l}\text { Experimenter- } \\
\text { delivered models }\end{array}$ & $\begin{array}{l}\text { Baseline and } \\
\text { Lag } 1\end{array}$ & $\begin{array}{l}\text { Naturalistic } \\
\text { Observation }\end{array}$ & Echoic \\
\hline Chloe & $\begin{array}{l}\mathrm{z}, \mathrm{t}, \mathrm{g}, \mathrm{oI}, \varepsilon, \mathrm{aI} \\
\text { BL1 only: d, w, I }\end{array}$ & $\begin{array}{l}\mathrm{z}, \mathrm{t}, \mathrm{g}, \mathrm{oI}, \varepsilon, \\
\text { aI, d, w, I }\end{array}$ & $\mathrm{t}, \mathrm{g}, \varepsilon, \mathrm{d}, \mathrm{w}$ & $\varepsilon, \mathrm{aI}, \mathrm{d}, \mathrm{w}, \mathrm{I}$ \\
\hline Ari & $\mathrm{b}, \mathrm{m}, \mathrm{w}, \mathrm{u}, \mathrm{i}, \mathrm{a}$ & $\mathrm{m}, \mathrm{w}, \mathrm{u}, \mathrm{i}, \mathrm{a}$ & $\mathrm{b}, \mathrm{m}, \mathrm{w}, \mathrm{u}, \mathrm{i}, \mathrm{a}$ & $\mathrm{m}, \mathrm{w}, \mathrm{u}$ \\
\hline Lily & $\mathrm{g}, \mathrm{t}, \mathrm{h}, \mathrm{u}, \Lambda, \mathrm{i}$ & $\mathrm{g}, \mathrm{t}, \mathrm{h}, \mathrm{u}, \Lambda, \mathrm{i}$ & $t, h, v, i$ & $\Lambda$ \\
\hline
\end{tabular}

and hee. Models were presented in a semirandom order; which was predetermined and included on the data sheet. All nine vocal models included in the set were presented in three consecutive sessions before any model was repeated. All models were presented an equal number of times across all sessions.

Table 1 lists the consonants and vowels used in the vocal models for each participant. The vocal models for Chloe were changed after the first four baseline sessions because Chloe frequently echoed three of the phonemes. The phonemes that Chloe echoed most frequently (" d,", "w," and “'ih") were replaced with " $z$,", "t,", and "oy" following consultation with the SLP.

General procedure. Immediately prior to each session, the experimenter brought the participant to the work area, gained his or her attention, and implemented an MSWO preference assessment to determine the stimuli to be used as reinforcers during the session. Prior to the first session of the day and between each session, a 1-min to 3-min break was provided, during which the experimenter provided attention and noncontingent access to play materials.

Each session consisted of six trials. The experimenter initiated each trial by gaining the participant's attention and presenting a vocal model. The experimenter provided a $15 \mathrm{~s}$ opportunity to respond following the vocal model. The purpose of the $15 \mathrm{~s}$ interval was to allow repeated or varied responses sufficient time to contact the prevailing contingencies during the experimental phases (described below). The experimenter delivered a reinforcer within $3 \mathrm{~s}$ if the participants engaged in phonemes that were eligible for reinforcement according to the schedule currently in effect. The reinforcement interval lasted either $30 \mathrm{~s}$ or until the small edible reinforcer was consumed. After the reinforcement interval, the experimenter gained the participant's attention and presented the next vocal model. If the participant did not emit a phoneme eligible for reinforcement within $15 \mathrm{~s}$, the experimenter initiated the next trial by presenting another vocal model.

The procedure was modified for Ari due to high levels of stereotypy and difficulties with gaining his attention. Immediately prior to the presentation of each vocal model, the experimenter delivered an attending prompt followed by two mastered gross motor imitation tasks. The attending prompt consisted of the experimenter (a) placing her pointer finger to her lips while delivering the instruction "quiet," (b) waiting until Ari placed his pointer finger to his lips, (c) stating "get ready" while placing her hands in her lap, and (d) waiting until Ari placed his hands in his lap. If at any point during the attending prompt, Ari vocalized, broke eye contact, or did not engage in the gestural components, the attending prompt was reinitiated. After gaining Ari's attention using the attending prompt, the experimenter immediately delivered the instruction "do this" while modeling a gross motor arm-and-hand movement (e.g., clap, wave). If Ari imitated the action correctly without engaging in vocal stereotypy, a second arm-and-hand gross motor imitation was presented with the instruction "do this." If Ari correctly imitated the second movement without vocal stereotypy, the vocal model was immediately presented. If Ari did not imitate either gross motor movement correctly without vocal stereotypy, the experimenter repeated the 
procedure beginning with the attending prompt. The attending procedure was rarely restarted with Ari and was never restarted more than once on any given trial.

Continuous reinforcement (CRF). During baseline sessions, the first phoneme that occurred within $15 \mathrm{~s}$ was reinforced on a CRF schedule (i.e., FR 1).

Lag 1 reinforcement schedule. During Lag 1 reinforcement schedule sessions, the participants' first phoneme during the first trial was reinforced and served as a base response to which to compare responses in subsequent trials. On the next trial, the experimenter reinforced the first phoneme that varied from the previous response (i.e., containing a phoneme not included in the previous response) during the 15 -s response interval. Additional criteria for reinforcement were added for Chloe and Ari. Due to the large number of vowels and diphthongs that Chloe was able to imitate during her pre-assessments (11 out of 14), only consonants and the modeled diphthongs and vowels ("aI," "oy," "eh") were eligible for reinforcement. That is, if a response varied from the previous response but no consonants or modeled vowels were different (e.g., "boo" and "bee") the response was not reinforced nor recorded as a different response. Therefore, only consonants and modeled vowels or diphthongs were included in the cumulative data collected during the second baseline and Lag 1 intervention phases for Chloe. Due to the difficulty of distinguishing phonemes that were embedded in Ari's vocal stereotypy, only phonemes that were emitted following 2 s without stereotypy were considered eligible for reinforcement according to the Lag 1 schedule.

Extinction of stereotyped responses (Chloe only). An extinction phase was implemented for Chloe after no new phonemes had been emitted for eight sessions and variability was at $0 \%$ for six of those sessions. Chloe emitted the phoneme " $w$ " during $100 \%$ of trials for every session and it comprised $90 \%$ of the reinforced phonemes (consonant or modeled diphthongs and vowels) emitted during the last session. Therefore, the phoneme " $\mathrm{w}$ " was placed on extinction for all further sessions. That is, responses were not reinforced that differed from the previous response only in that they included the phoneme "w." However, if the phoneme " $w$ " occurred in conjunction with another phoneme not included in the previous response, the response was reinforced. Otherwise, the same Lag-1 reinforcement procedures were still in effect.

Termination criteria. A minimum of 12 sessions was conducted with all participants in the Lag-1 phase. Intervention was discontinued for Chloe following 5 extinction sessions in which no novel phonemes were emitted and variability did not increase. Following the completion of intervention for Chloe, the criteria for discontinuation of intervention were changed from 12 sessions with no novel phonemes to 5 sessions across two days with no novel phonemes. The criteria were altered because Chloe engaged in mild escape behaviors (whining, attempting to leave). Intervention was discontinued for Ari and Lily according to the new criteria.

\section{RESULTS}

\section{Pre- and Post-Intervention Assessments}

Table 2 shows the results of the pre- and post-intervention assessments for each participant. The first and second data columns display the number of different English phonemes emitted during the pre- and postintervention naturalistic observation. The maximum score for the observation was 40 (i.e., the number of English phonemes). The number of different phonemes increased for all participants. The third and fourth data columns of Table 2 show the results of the EESA. The scores on the EESA increased for all participants; however, the magnitude of change for Ari and Lily was minimal. The fifth and sixth data columns of Table 2 show the pre- and post-intervention scores for the Level 1 Spontaneous Vocal Behavior subsection of the VB-MAPP. The Level 1 scores increased for Chloe and Lily. No change in scores was observed for Ari. The seventh and eighth data columns display the number of English phonemes echoed during the preand post-intervention echoic assessments. The maximum score for the echoic assessment was 40 (i.e., the number of English phonemes). Echoic responses increased by at least $100 \%$ for all participants. 
Table 2

Results of the pre-and post-intervention assessments for each participant. The maximum score for the naturalistic observation, EESA, VB-MAPP, and echoic assessment was 40, 25, 5, and 40, respectively

\begin{tabular}{|c|c|c|c|c|c|c|c|c|}
\hline \multirow[b]{2}{*}{ Participant } & \multicolumn{2}{|c|}{ Naturalistic Observation } & \multicolumn{2}{|c|}{ EESA } & \multicolumn{2}{|c|}{ VB-MAPP } & \multicolumn{2}{|c|}{ Echoic Assessment } \\
\hline & Pre & Post & Pre & Post & Pre & Post & Pre & Post \\
\hline Chloe & 6 & 23 & 7 & 18.5 & 2 & 4 & 13 & 26 \\
\hline Ari & 15 & 25 & 1 & 1.5 & 3 & 3 & 2 & 5 \\
\hline Lily & 11 & 22 & 8 & 10.5 & 2 & 3 & 7 & 14 \\
\hline
\end{tabular}

Figure 1 shows the frequency of each of the English phonemes emitted by the participants during the 1-hr pre- and post-intervention naturalistic observations. The variety and the frequency of phonemes increased during the post-intervention assessments for all participants.

\section{Intervention}

Figure 2 displays the cumulative number of novel phonemes emitted by each participant across the CRF baseline, Lag 1, and Lag 1 plus extinction (Chloe only) phases. The number contained within the parentheses represents the cumulative number of novel phonemes participants emitted within each phase. For all participants, the number of novel phonemes increased during the CRF baseline and the Lag-1 phase. For Chloe, no novel phonemes occurred during the Lag 1 plus extinction for " $w$ " phase.

The first data column of Table 3 shows the number of new phonemes (i.e., phonemes that did not occur during the pre-intervention 1-hr naturalistic observation and echoic assessment) that occurred during the CRF baseline and Lag 1 phases. The second and third data columns display the proportion of the new phonemes observed during the baseline and Lag 1 phases that also occurred during the post-intervention observation and echoic assessment. For all participants, about half of the new phonemes that occurred during the CRF baseline and Lag 1 phase occurred during the post-intervention naturalistic observation. A smaller proportion of new phonemes were echoed during the postintervention echoic assessment.

Figure 3 displays an index of response variability during the $\mathrm{CRF}$ baseline and Lag
1 phases for each participant. The percentage of trials with variability was calculated by dividing the number of trials in which the primary data collector coded " $D$ "' (i.e., a different response) by the total number of trials and multiplying by 100 . For Chloe's evaluation (top panel of Figure 3), the percentage of trials with variability in responses averaged $50 \%, 47 \%, 18 \%$, and $24 \%$ of trials during the baseline 1 , baseline 2, and Lag 1, and Lag 1 plus extinction for "w" phases, respectively. Chloe frequently emitted the same phonemes in the same order on multiple trials during intervention ("wuhwee"). During the "w" extinction phase, Chloe repeatedly emitted another sequence of phonemes "oo-ee." This response may have functioned as a mand to access a preferred activity (DVD player and DVDs), which was not present during experimental sessions. For Ari's evaluation (middle panel of Figure 3), the percentage of trials with response variability averaged $46 \%$ and $80 \%$ during the baseline and Lag 1 phases, respectively. For Lily's evaluation (bottom panel of Figure 3), the percentage of trials with response variability averaged $46 \%$ and $86 \%$ during the baseline and Lag 1 phases, respectively.

Table 1 lists the experimenter-delivered vocal models for each participant and which of the phonemes that served as vocal models were emitted during experimental sessions (i.e., baseline and Lag 1 phases) and in the post-intervention observational and echoic assessments. None of the modeled phonemes for any participant were emitted during preintervention assessments. During the baseline and Lag 1 phases, the participants emitted all or most of the modeled phonemes. Although less consistent, all participants emitted a proportion of the modeled phonemes during 

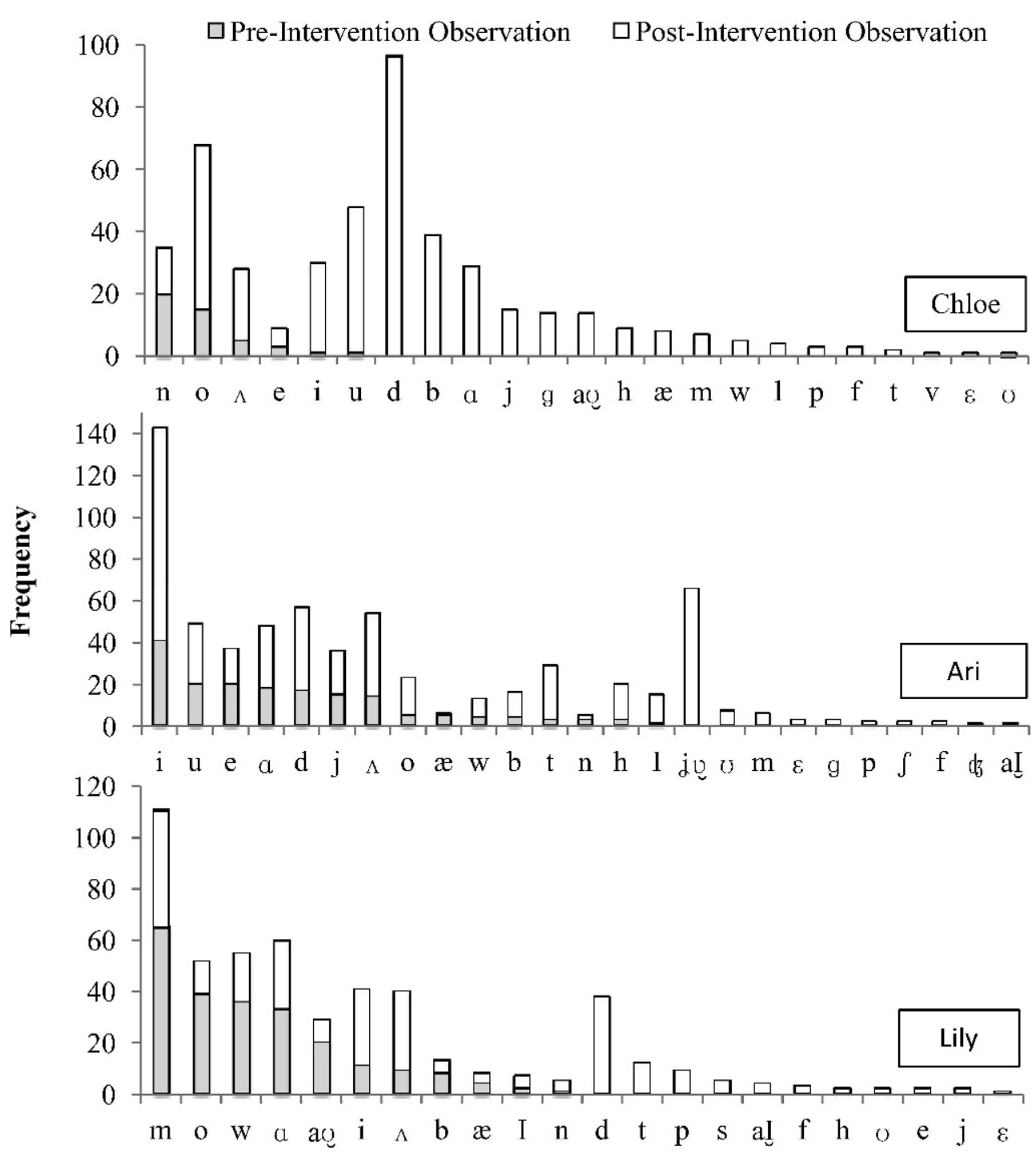

\section{English Phonemes}

Figure 1. The stacked frequency of each English phoneme during the 1-hr pre- and post-intervention naturalistic observations.

the post-intervention naturalistic observation and echoic assessment.

Transcriptions of the sessions were reviewed following the completion of intervention to determine if other forms of novel vocal behavior (e.g., novel combinations of phonemes, echoic behavior, word approximations) emerged during the final sessions in which no novel phonemes were emitted. For all participants, no novel vocal behaviors were observed in the final experimental sessions.

\section{DISCUSSION}

The current study provides a partial replication of the results reported by Esch et al. (2009) supporting the use of lag reinforcement schedules to increase vocal variability in nonverbal children with little to 


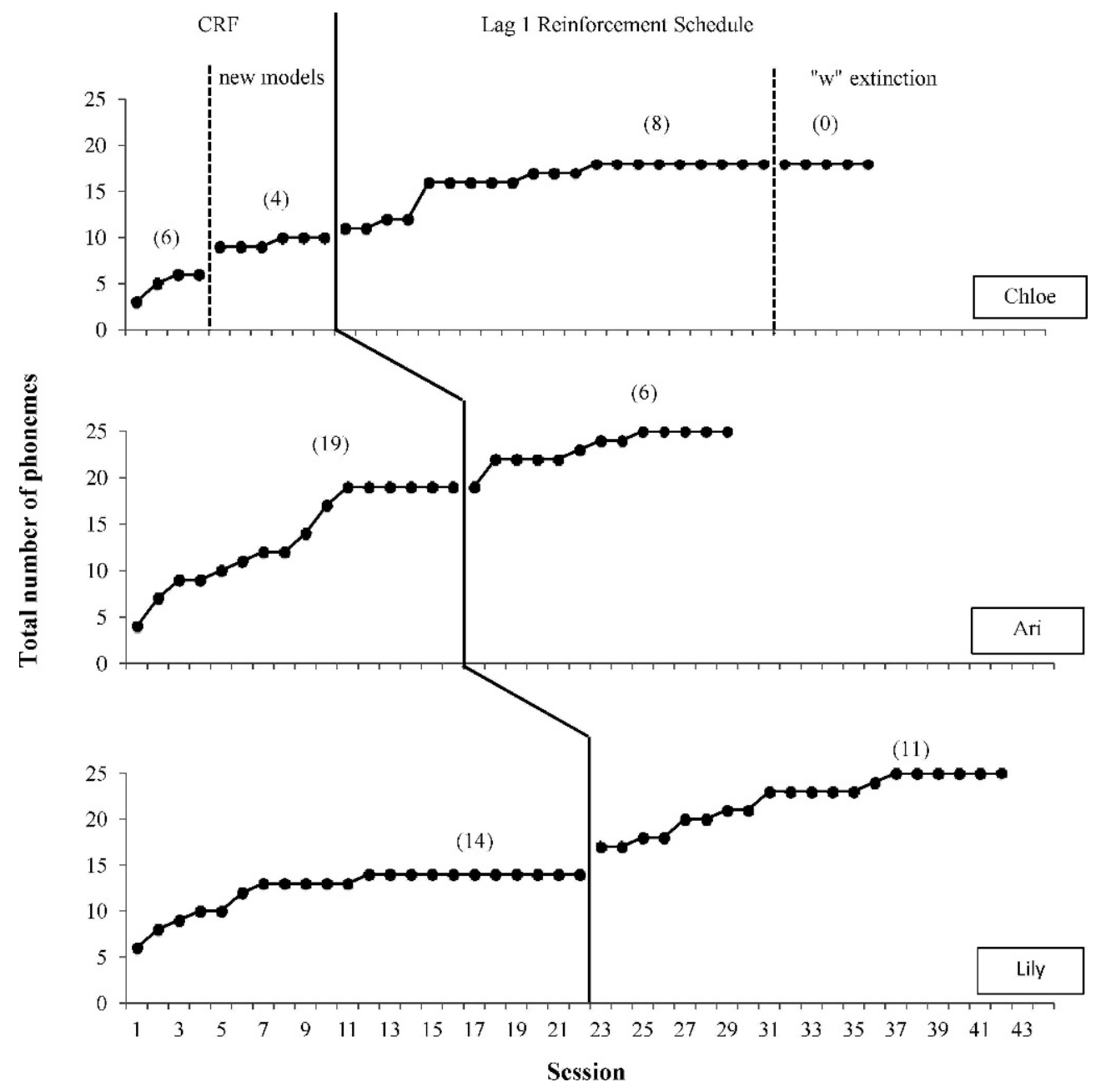

Figure 2. The cumulative number of novel phonemes emitted by each participant across CRF, Lag 1, and Lag 1 plus extinction conditions. The cumulative number of novel phonemes emitted within each phase is represented in parentheses.

no echoic repertoire. This study extends the research by Esch et al. through a number of procedural differences. As in the study by Napolitano et al. (2010), we attempted to define variability in a socially and clinically significant manner. While the short-term goal of the Lag 1 intervention was to increase variability, the long-term goal was to increase the variety of phonemes in the participant's repertoire to prepare them for functional vocal language training. After an individual has acquired a variety of phonemes, instructors can use shaping to bring vocalizations under stimulus control as verbal operants (e.g., echoics, mands, tacts).
To this end, the definition of variability specified the inclusion of a different phoneme than the previously emitted phoneme and excluded differences among vocalizations based only on the sequence of phonemes. Another difference is that we collected data on the cumulative number of novel phonemes emitted by each participant throughout the study. As in the study by Lee et al. (2002), the cumulative novel response data showed that participants emitted novel vocal behavior during the Lag 1 phase, supporting the use of the lag schedule to generate new phonemes. If variability increased but novel phonemes did not, the 
Table 3

The number of new phonemes during intervention and the proportion of new phonemes observed during the postintervention naturalistic observation (NO) and echoic assessment

\begin{tabular}{lccc}
\hline & & \multicolumn{2}{c}{$\begin{array}{c}\text { Post-intervention } \\
\text { Assessments }\end{array}$} \\
\cline { 3 - 4 } Participant & Intervention & NO & Echoic \\
\hline Chloe & 13 & $8 / 13$ & $7 / 13$ \\
Ari & 12 & $8 / 12$ & $2 / 12$ \\
Lily & 12 & $8 / 12$ & $2 / 12$ \\
\hline
\end{tabular}

participants might have been alternating between existing responses, which is counterproductive for the purposes of the current study. That is, increasing variability without expanding the repertoire of phonemes does not prepare the participant for further vocal training.

Baseline consisted of delivering reinforcers on a CRF (i.e., FR1) schedule for all phonemes. Initially, the cumulative number of novel phonemes increased for all participants. For both Chloe and Lily, variability early in the CRF phase was relatively high. As the participants' phonemes gained a history with the CRF schedule, variability decreased and a plateau was reached in which no novel phonemes were emitted. It is possible that the number of novel phonemes would have increased in the absence of implementing the Lag 1 schedule. However, the longer plateaus observed for Ari (5 sessions) and Lily (10 sessions) support the prediction that evoking additional novel phonemes during CRF conditions was unlikely.

The current study has limitations that warrant discussion. First, the results were somewhat inconsistent across participants suggesting the procedure may not be equally effective or efficient with all children diagnosed with autism spectrum disorders. Further replication with additional participants is needed. Second, changes in postintervention assessment scores cannot be directly attributed to the intervention, although occurrence of novel phonemes first emitted during intervention suggest a potential effect. Third, the resources required to implement the data collection system for this study were extensive. The first author completed $40 \mathrm{hrs}$ of transcription practice to establish acceptable interobserver agreement with a trained SLP on the participants' phonemes. In addition, the procedure required videotaping and transcribing sessions to track the emergence of phonemes. The less-thanideal interobserver agreement scores obtained in the study may relate to challenges with transcribing phonemes. Many English phonemes can be difficult to distinguish from each other (e.g., "ah" and "uh"), particularly when emitted outside of the context of functional vocal verbal behavior. Transcription and interobserver reliability were particularly difficult for Ari due high levels of stereotypy. For future studies, researchers may focus on measuring the percentage of trials with variability, because variability can be measured relatively simply by recording responses using codes (i.e., N, R, or D) to reduce the complexity of the measurement system. Another possibility is for experimenters to track novel phonemes during sessions and keep an ongoing list of cumulative novel phonemes.

The results of this study suggest that lag schedules of reinforcement for phonemes enhanced the echoic control for some individuals. Echoic responses were incidentally reinforced on occasion during the Lag 1 sessions. That is, if the participant's response included a phoneme that was modeled and the response included a phoneme that was different from the previous response, the response was reinforced. Throughout the study, the participant's behavior was reinforced regardless of whether the response shared point-to-point correspondence with the experimenter-delivered vocal model. It is possible that future echoic training may be more difficult if a learner has a history of gaining access to reinforcers for emitting phonemes that differ from the vocal models. Vocal models were included during experimental sessions as part of replicating the Esch et al. (2009) study. Researchers might consider omitting the experimenter-delivered vocal models to circumvent potential hindrances to future echoic training.

The current study and Esch et al. (2009) were conducted in an analog setting (e.g., therapy rooms, unused conference room) using a complex data collection system. Future research should explore variations of 


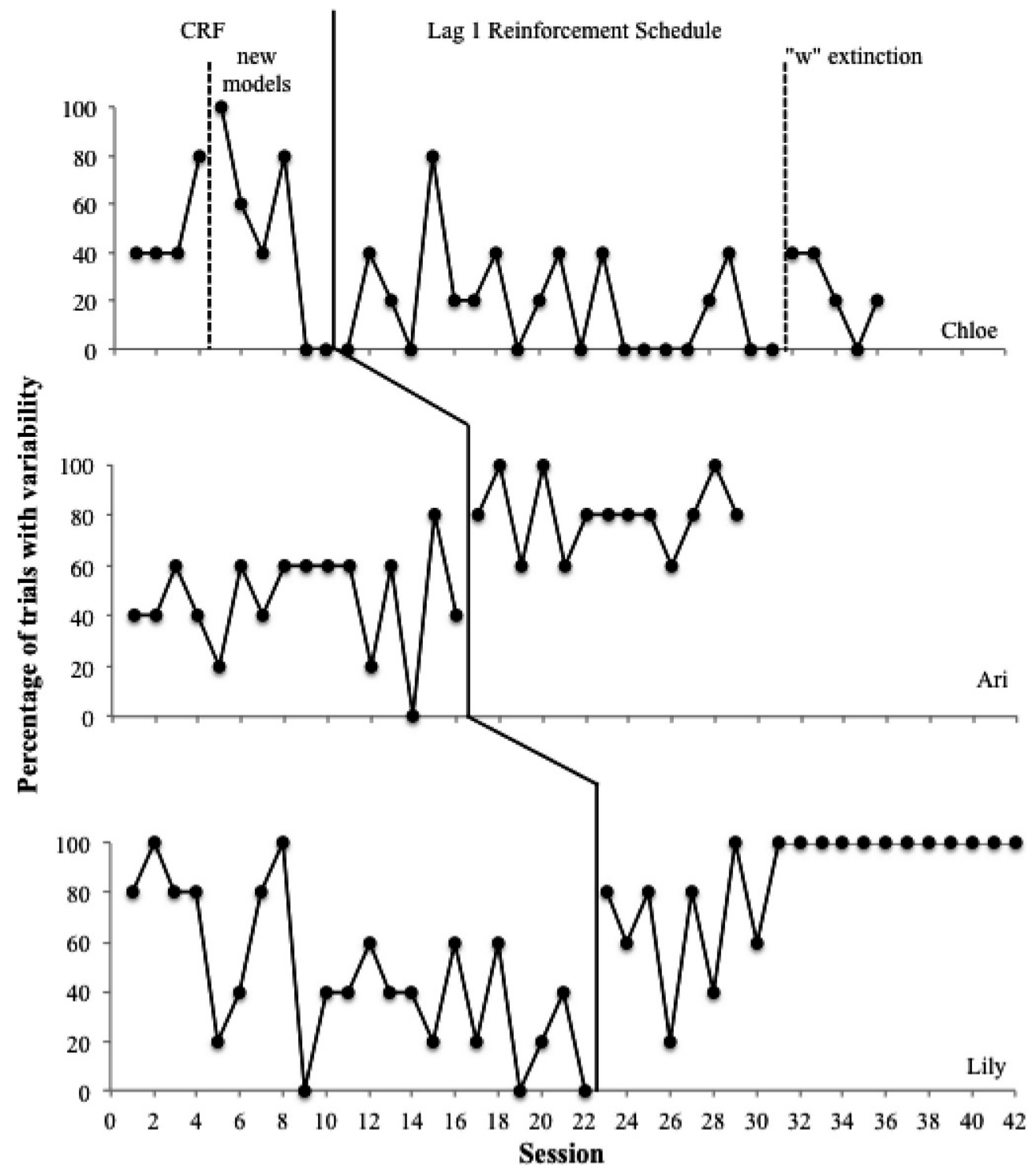

Figure 3. The percentage of trials with variability during continuous reinforcement (CRF), Lag 1, and extinction phases for each participant.

the procedure to increase the clinical utility of the lag schedule. Researchers may consider conducting sessions during naturally occurring play with caregivers or familiar adults using a less complex data collection system.

\section{REFERENCES}

American Psychiatric Association. (2000). Diagnostic and statistical manual of mental disorders (4th ed., text revision). Washington, DC: Author.
DeLeon, I. G., \& Iwata, B. A. (1996). Evaluation of multiple-stimulus presentation format for assessing reinforcer preferences. Journal of Applied Behavior Analysis, 29, 519-533.

Duker, P. C., \& van Lent, C. (1991). Inducing variability in communicative gestures used by severely retarded individuals. Journal of Applied Behavior Analysis, 24, 379-386.

Esch, J. W., Esch, B. E., \& Love, J. R. (2009). Increasing vocal variability in 
children with autism using a lag schedule of reinforcement. The Analysis of Verbal Behavior, 25, 73-78.

Fisher, W., Piazza, C. C., Bowman, L. G., Hagopian, L. P., Owens, J. C., \& Slevin, I. (1992). A comparison of two approaches for identifying reinforcers for persons with severe and profound disabilities. Journal of Applied Behavior Analysis, 25, 491-498.

Leaf, R., \& McEachin, J. (1999). A work in progress: behavior management strategies and a curriculum for intensive behavioral treatment of autism. New York, NY: DRL Books.

Lee, R., McComas, J. J., \& Jawor, J. (2002). The effects of differential and lag reinforcement schedules on varied verbal responding by individuals with autism. Journal of Applied Behavior Analysis, 35, 391-402.
Lovaas, O. I. (2003). Teaching individuals with developmental delays: Basic intervention techniques. Austin, TX: Pro-Ed.

Napolitano, D. A., Smith, T., Zarcone, J. R., Goodkin, K., \& McAdam, D. B. (2010). Increasing response diversity in children with autism. Journal of Applied Behavior Analysis, 43, 265-271.

Skinner, B. F. (1957). Verbal behavior. Englewood Cliffs, NJ: Prentice-Hall.

Sundberg, M. L. (2008). Verbal behavior milestones assessment and placement program. Concord, CA: AVB Press.

Watson, P. J., \& Workman, E. A. (1981). The non-concurrent multiple baseline acrossindividuals design: An extension of the traditional multiple baseline design. Journal of Behavior Therapy and Experimental Psychiatry, 12, 257-259. 